\title{
PRODUCTION OF SUBMICRONSTRUCTURES IN HIGHLY CONDUCTIVE SUBSTRATES
}

\author{
H. A. M. VAN DEN BERG
}

Delft University of Technology, The Netherlands

\begin{abstract}
Résumé. - On a étudié la technique de projection optique sur des substrats de réflectivité élevée, tels que des métaux, afin de préparer des dessins submicrométriques. La formation d'images avec des objectifs à ouverture élevée a été traitée théoriquement. Le caractère vectoriel du rayonnement électromagnétique a été entièrement pris en considération. Des conclusions ont été tirées quant à l'image dans la laque photosensible et au dessin formé dans le métal par gravure chimique ou par gravure par bombardement ionique. On montre, aussi bien théoriquement qu'expérimentalement, que les problèmes, associés avec les ondes stationnaires, peuvent être résolus par application d'une couche anti-reflet de silicium ou sélénium sur les couches métalliques.
\end{abstract}

Abstract. - The optical projection technique on highly reflective substrates has been considered in order to realize patterns far into the submicron range. The image formation utilizing high numerical lenses has been theoretically treated. In the section on theory full account has been given of the vectorial character of the electromagnetic radiation. Conclusions have been drawn regarding the image in the resist and about the pattern which is formed in the metal if chemical or sputter etching is applied. It is shown theoretically as well as experimentally that the problems arising in conjunction with the standing waves can be solved by applying anti-reflecting coatings consisting of amorphous silicon and selenium.

1. Introduction. - Up to now optical techniques such as contact printing and optical projection lithography have been used everywhere in the world to realize devices with smallest dimensions in the submicron range. The high reflectivity of the metals introduces standing waves in the resist, as was distinguished by S. Middelhoek [1] in the late sixties. A number of investigators have studied the related phenomena [2, 3, 4], mostly in relation to contact printing or to situations in which objectives with a very low numerical aperture are used.

In this paper a theoretical treatment will be given of the image formation by objectives with an arbitrary numerical aperture on highly reflective substrates. In this theory full account has to be given of the vectorial character of the electromagnetic radiation. It is well known, that the problems arising in conjunction with the standing waves can be alleviated by supplying the substrates with an anti-reflection coating of a dielectric with a high reflective index such as $\mathrm{Cr}_{2} \mathrm{O}_{3}$ and $\mathrm{Fe}_{2} \mathrm{O}_{3}$. However, especially in the case of very high reflective substrates, there is a great need of materials to suppress the standing waves.

2. Theory. -2.1 IMAgE FORMATION ON HIGHLY REFLECTIVE SURFACES. - With respect to the image formation by projection lithography a number of papers have been written which are based on the scalar diffraction theory $[5,6]$. Such a theoretical treatment is only meaningful if the refractive indices of the substrate and the resist do not deviate sharply and if the numerical aperture of the objective is relatively low. The tangential components of the electric field are forced to zero at the metal-resist boundary if the reflectivity of the metal is high. In

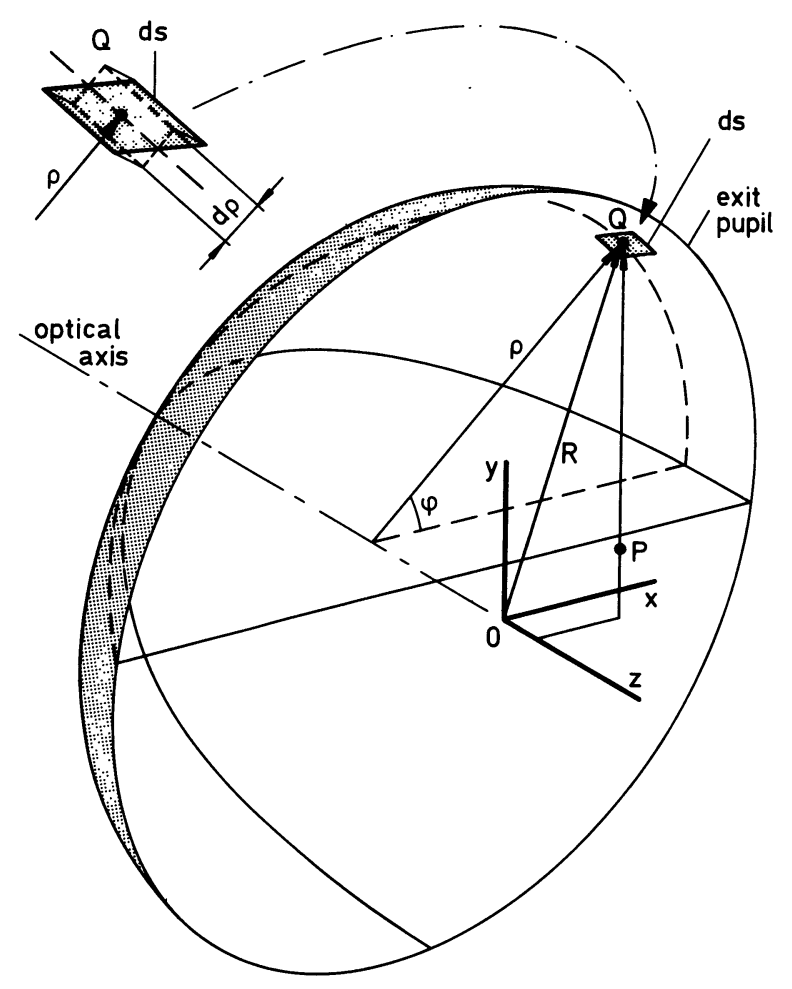

Fig. 1. - The spherically secondary-source surface at the exit pupil contributing to the image of a point in a homogeneous space. 
this situation, only a theory based on the vectorial character of the electromagnetic radiation can be of help. Wolf and coworkers [7, 8, 9] have treated the near-focus energy-density distribution of a polarized plane wave focussed by an aplanatic lens system. The calculations presented in this paper are based on a slightly different approach. The spherical surface which has its centre in the Gaussian image point serves as a secondary source. In this treatment, which is very similar to the Fresnel-Kirchhoff approach, only that part of the sphere that is not obstructed by the exit pupil (see Fig. 1) contributes to the image. The influence of the electrically perfect conducting screen is represented by the mirror-source of the secondary source surface which is symmetrically positioned with respect to the screen. We consider the image of a point from an object in the object space which has been illuminated by a linear polarized plane wave. The image of the total object can be deduced from the image of a point by integration over all point sources in the object contributing to the image. It is illustrative to distinguish two extreme situations. In the case of coherent illumination the amplitude distribution of the point sources have to be added in the right phase to obtain the amplitude in a specific point of the image. In contrast the intensity at a certain position due to a mutual incoherent illuminated object is found by integrating over the intensity of the contributions of all point sources.

The details of the lengthy calculation and the justification of the theory can be found elsewhere [10]. For the image of a point which is illuminated by a monochromatic plane wave, which is polarized along the $y$-axis, it can be derived :

$$
\begin{aligned}
& E_{x}\left(x^{\prime}, y^{\prime}, z^{\prime}\right)=\int_{0}^{N . A .}+A . J_{2}(h) \frac{x^{\prime} y^{\prime}}{x^{\prime 2}+y^{\prime 2}} \cdot \sin \left\{k R\left(1-\rho^{\prime 2}\right)^{1 / 2} z^{\prime}\right\} \mathrm{d} \rho^{\prime} \\
& E_{y}\left(x^{\prime}, y^{\prime}, z^{\prime}\right)=\int_{0}^{N . A .}\left[D . J_{0}(h)+E . J_{2}(h) \cdot \frac{y^{\prime 2}-x^{\prime 2}}{x^{\prime 2}+y^{\prime 2}}\right] \sin \left\{k R\left(1-\rho^{\prime 2}\right)^{1 / 2} z^{\prime}\right\} \mathrm{d} \rho^{\prime} \\
& E_{z}\left(x^{\prime}, y^{\prime}, z^{\prime}\right)=\int_{0}^{N . A .} B . J_{1}(h) \cdot \frac{y^{\prime}}{\left(x^{\prime 2}+y^{\prime 2}\right)^{1 / 2}} \cdot \cos \left\{k R\left(1-\rho^{\prime 2}\right)^{1 / 2} z^{\prime}\right\} \mathrm{d} \rho^{\prime}
\end{aligned}
$$

where :

$N . A$. is the numerical aperture of the objective,

$x^{\prime}, y^{\prime}, z^{\prime}, \rho^{\prime}$ are $x / R, y / R, z / R$, and $\rho / R$ respectively and are defined by figure 1 ,

$d^{\prime}$ is $d / R$ where $d$ is the distance of the Gaussian image point to the screen,

$h$ is $k R \rho^{\prime}\left(x^{\prime 2}+y^{\prime 2}\right)^{1 / 2}$,

$k$ is the magnitude of the wave-vector $2 \pi / \lambda$,

$A$ is $i 4 \pi C^{\prime} \frac{-1+\left(1-\rho^{\prime 2}\right)^{1 / 2}}{\left(1-\rho^{\prime 2}\right)^{1 / 4}} \rho^{\prime} \mathrm{e}^{-i k R d^{\prime}\left(1-\rho^{\prime 2}\right)^{1 / 2}}$,

$B \quad$ is $i 4 \pi C^{\prime} \frac{\rho^{\prime}}{\left(1-\rho^{\prime 2}\right)^{1 / 4}} \rho^{\prime} \mathrm{e}^{-i k R d^{\prime}\left(1-\rho^{\prime 2}\right)^{1 / 2}}$,

$D$ is $-2 \pi i C^{\prime} \frac{1+\left(1-\rho^{\prime 2}\right)^{1 / 2}}{\left(1-\rho^{\prime 2}\right)^{1 / 4}} \rho^{\prime} \mathrm{e}^{-i k R d^{\prime}\left(1-\rho^{\prime 2}\right)^{1 / 2}}$,

$E$ is $-2 \pi i C^{\prime} \frac{1-\left(1-\rho^{\prime 2}\right)^{1 / 2}}{\left(1-\rho^{\prime 2}\right)^{1 / 4}} \rho^{\prime} \mathrm{e}^{-i k R d^{\prime}\left(1-\rho^{\prime 2}\right)^{1 / 2}}$, and

$C^{\prime}$ represents the magnitude of object.

In figure 2 the contours of equal electric-energy density for a plane which coincides with the optical axis and the polarization vector have been plotted. This quantity determines which part of the photoresist is removed by the developer after the exposure. Figures 3 and 4 give the amplitude distribution in two directions and surfaces : at the metal-resist interface and at a distance of $\lambda / 4$ apart from the surface respectively. The standing wave character in the image is clearly demonstrated, and its destructive effect on the resulting pattern is self-evident.
2.2 The STANDing WAVES AND THE ANTI-REFLECTION COATINGS. - Until now no exhaustive treatment of anti-reflection coatings with only one single additive layer has been given. In a system, as shown in figure 5, absorbing layers must be allowed. Using the matrix method, such as is proposed by Heavens [11], the standing wave ratio for normal incident is derived and is given by :

$$
\left|\frac{E_{1}^{-}}{E_{1}^{+}}\right|=\left|\frac{\rho_{1,2}^{E}+\rho_{2,3}^{E} \cdot \exp \left(-2 \gamma_{2} h_{2}\right)}{1+\rho_{1,2}^{E} \cdot \rho_{2,3}^{E} \cdot \exp \left(-2 \gamma_{2} h_{2}\right)}\right|
$$




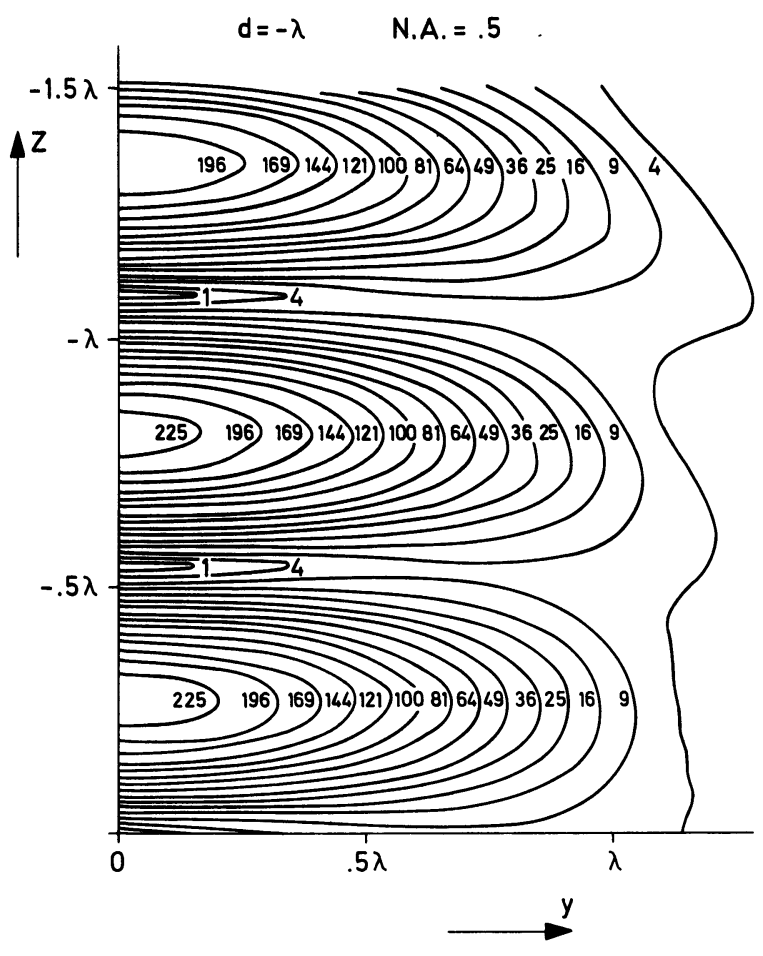

Fig. 2. - The image of a point. Contours of equal electric-energy density. $d=\lambda, N . A .=0.5$.

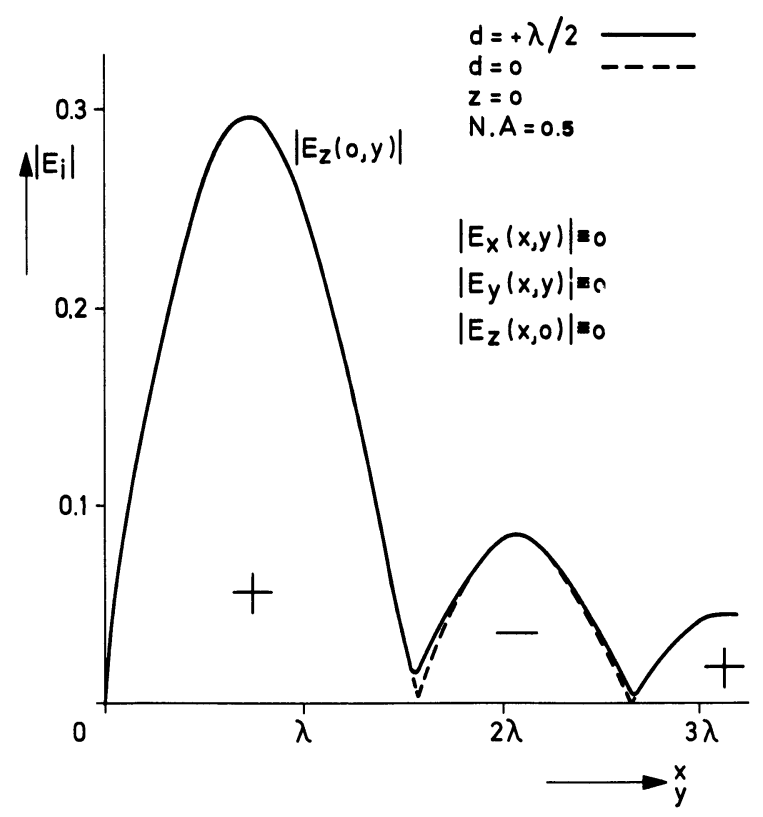

FIG. 3. - The intensity profiles of the image of a point at the metal surface, in the direction along the polarization vector and along a line normal to it. $d=\lambda / 2, N . A .=0.5$.

where

$$
\gamma_{2}=i \omega\left(\varepsilon_{0} \mu_{0}\right)^{1 / 2} \bar{n}_{2}
$$

and

$\rho_{0, p}^{E}=\left\{k_{0}-k_{\mathrm{p}}+i\left(n_{0}-n_{\mathrm{p}}\right)\right\} /\left\{k_{0}+k_{\mathrm{p}}+i\left(n_{0}+n_{\mathrm{p}}\right)\right\}$.

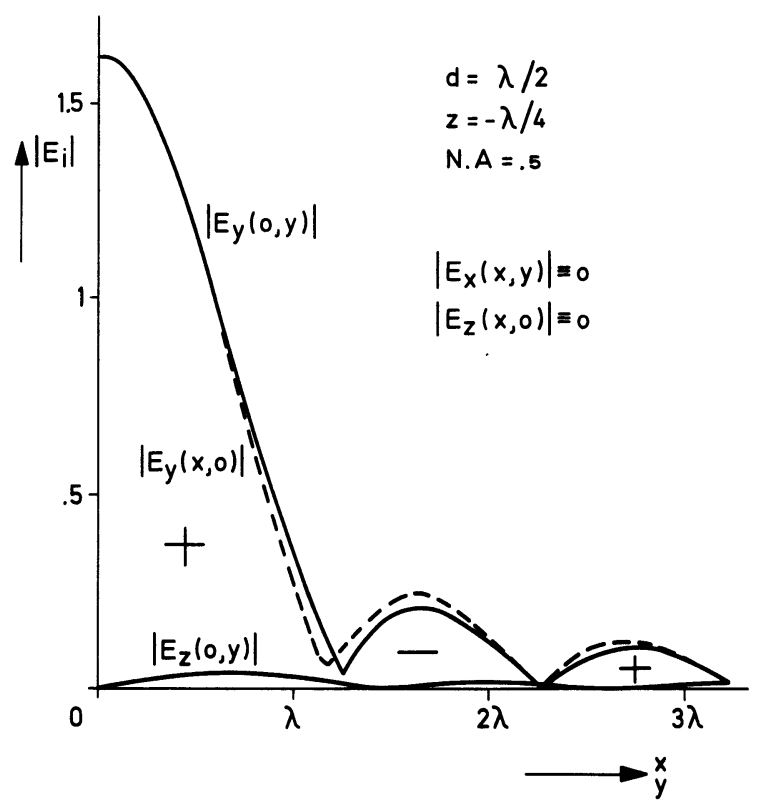

FIG. 4. - The intensity profiles of the image of a point $\lambda / 4$, apart from the reflecting screen, in the direction along the polarization vector and along a line normal to it. $d=\lambda / 2, N . A .=0.5$.

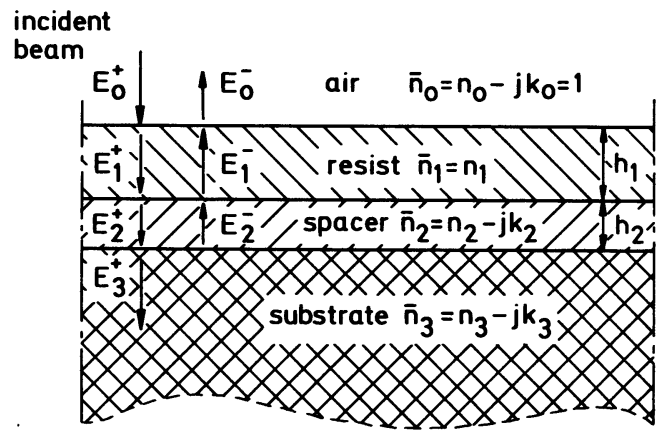

FIG. 5. - Cross-section of the layered structure, $E_{i}^{+}$is the amplitude of the wave propagating in the direction of the incident beam on the system, while $E_{i}^{-}$is the amplitude of the wave propagating in the opposite direction. $E_{0}^{+}=1, E_{3}^{-}=0$.

In this formula $E_{1}^{-}$and $E_{1}^{+}$are defined by figure 5 while $n_{\mathrm{p}}$ and $k_{\mathrm{p}}$ are the real and the imaginary part respectively of the refractive index $\bar{n}_{\mathrm{p}}$.

Of course the most desirable value of the standing wave ratio is zero. Since we are restricted to actually existing materials, there is no opportunity to accomplish this optimum in the practical situation.

A good standard for the quality of the system is given by the next expression :

$$
Q=\left|\frac{\left|E_{1}^{+}\right|+\left|E_{1}^{-}\right|}{\left|E_{1}^{+}\right|-\left|E_{1}^{-}\right|}\right|^{2} .
$$

The materials which best satisfy the demands will be found in the group of semiconductors with a relatively large bandgap. Amorphous silicon, selenium and selenium-arsenic compounds are especially 
suitable, due to the relatively large ratio of $n / k$. We selected permalloy, in connection with its great importance to magnetic bubble devices, to serve as an example. In figures 6 and 7 the $Q$ has been given as a function of the height $h_{2}$ of the spacer consisting of $\mathrm{Si}$ and $\mathrm{Cr}_{2} \mathrm{O}_{3}$ respectively.

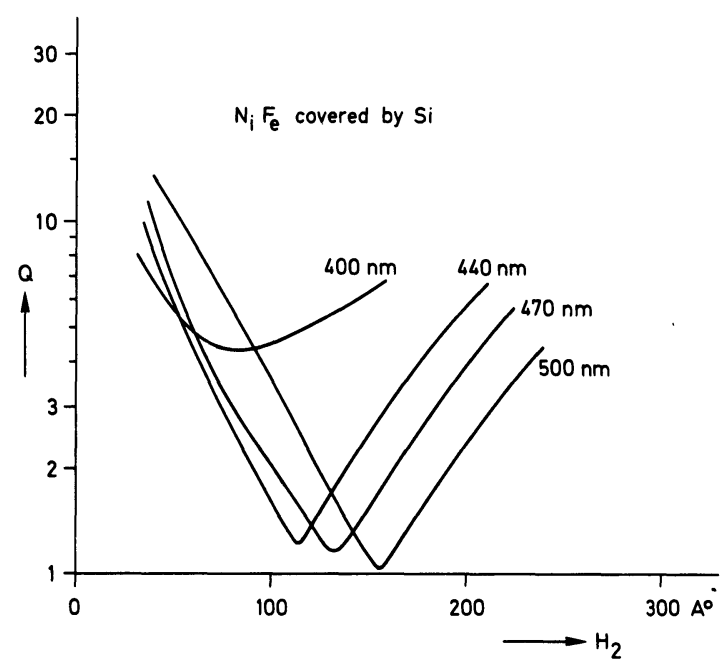

FIG. 6. - $\mathrm{NiFe}(80-20)$ supplied by $\mathrm{Si}$ with thickness $H_{2}$. The wavelength of the light source has been chosen as parameter (data [13]).

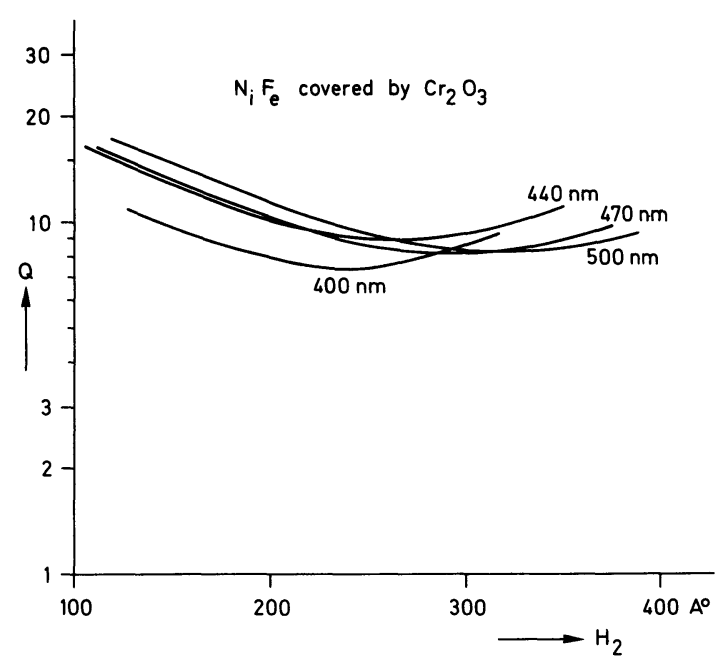

FIG. 7. - $\mathrm{NiFe}(80-20)$ supplied by $\mathrm{Cr}_{2} \mathrm{O}_{3}$. The wavelength of the light source has been chosen as parameter (data [13]).

3. Discussion. - The contours of equal electric energy density (Fig. 1) clearly demonstrate a periodic amplitude-variation along the optical axis. How closely this ideal profile can be approximated in practice depends on the reflectivity of the substrate and on the self-coherence of the light source. If the light source is quasi-monochromatic and the natural linewidth of the spectral lines which contribute to the exposure of the resist is relatively small, then the self coherence is high [12]. Since the application of glow discharge sources, is common practice in the optical photolithography, the interference effects will appear. From this it can be concluded that even when the mutual coherence at the penultimate mask (object) is zero, a situation which is often called incoherent illumination of the mask, a standing wave pattern will exist in the resist. Consequently a relatively low intensity level will be present at the resist-metal interface. The reduction of the intensity will be even more pronounced if the mutual coherence at the penultimate mask is high. The mutual coherence depends on the numerical aperture of the illumination system, if no coherent source such as a laserbeam is used [12].

In this case, in the centre of an area that corresponds to a transparent part of the penultimate mask, the neighbouring images of the point sources cancel at the metal surface, and the resulting intensity is zero (notice the point symmetry with respect to 0 of the amplitude profiles in figure 3). Only at the edges of the geometrical image can a resulting amplitude be expected. The exposed area at the surface will be concentrated predominantly under the opaque area of the geometrical image of the penultimate mask. If a thin resist layer is capable of preventing the specific chemical etch from attacking the metal, a completely deformed metal pattern will result.

Sputteretching of the pattern is frustrated by the undermined resist pattern at the edges, which causes degradation of the edge acuity. Consequently it is of great importance to supply it with an anti-reflection coating. Selenium and silicon.will be very profitable. Figure 6 proves that silicon needs a low-pass filter (e.g. most glasses) to exclude the U.V. radiation while selenium needs no filter at all. We have tried out these materials on several substrates such as chromium, $\mathrm{NiFe}$ and aluminium [13]. A remarkable reduction of the exposure time of a factor of more than 10 can be observed, and the range over which the exposure time could be varied without affecting the patterns increases enormously. The effect of $\mathrm{Cr}_{2} \mathrm{O}_{3}$ on the quality factor $Q$ is much less pronounced. For all that, it can be benefitially used by shifting the critical resist-oxide boundary to a desirable position in the standing wave pattern. Figure 4 proves that at a height of $\lambda / 4$ from the metal surface (about $440 \AA$ at the $436 \mathrm{~nm} \mathrm{Hg}$-line), the intensity profile is very similar to the ideal image of a point well known from scalar diffraction theory. From figure 7 it can be seen that the optimum $\mathrm{Cr}_{2} \mathrm{O}_{3}$ thickness on $\mathrm{NiFe}$ from an optical point of view is $260 \AA$. In practice a compromise is used, and it has even been shown that by using chemical etching, patterns can be realized with smallest dimensions of $0.2 \mu \mathrm{m}$ [14]. Nothwithstanding these good results with $\mathrm{Cr}_{2} \mathrm{O}_{3}$, a better defined pattern, which is less sensitive to process variations, can be realized by using $\mathrm{Si}$ and $\mathrm{Se}$.

Acknowledgments. - The author is greatly indebted to Prof. dr. ir. S. Middelhoek for stimulating discussions on the subject. He also wishes to thank J. B. van Staden for the technical assistance. 


\section{References}

[1] Middelhoek, S., I.B.M. J. Res. Develop. 14 (1970) 1077.

[2] Neureuther, A. R. and Dill, F. H., Proc. Microwave Res. Inst. Symp. XXIII (1974) 223.

[3] Widmann, D. W., Appl. Opt. 14 (1975) 931.

[4] Heitman, W. G. and van den Berg, P. M., Can. J. Phys. 53 (1975) 1305.

[5] Berg, H. A. M. van den and Ruigrok, J. J. M., Appl. Phys. 16 (1978) 279.

[6] Rowe, S. H., Opt. Soc. Amer. 59 (1969) 711.

[7] Wolf, E., Proc. Roy. Soc. (London) A 253 (1959) 349.

[8] Boivin, A., Dow, J. and Wolf, E., J. Opt. Soc. Amer. 57 (1967) 1171.
[9] Richards, B. and Wolf, E., Proc. Roy. Soc. (London) A 253 (1959) 358.

[10] BERG, H. A. M. van DEN, J. Opt. Soc. of Amer. to be published.

[11] Heavens, O. S., Thin Film Physics (Methuess \& Co Ltd London) 1970.

[12] Born, M. and Wolf, E., Principles of optics (4th Pergamon Press) 1975.

[13] Berg, H. A. M. van den and Staden, J. B. van, J. Appl. Phys. to be published.

[14] Berg, H. A. M. van DEN, Humphryes, R. F., RuigroK, J. J. M. and Venema, A., Proc. Ultrason. Symp. (1977) 767. 